\title{
BMJ Open Impact of child summertime obesity interventions on body mass index, and weight-related behaviours: a systematic review and meta-analysis protocol
}

\author{
Jennette P Moreno, ${ }^{1}$ Lydi-Anne Vézina-Im, ${ }^{1}$ Elizabeth M Vaughan, ${ }^{2}$ \\ Tom Baranowski ${ }^{1}$
}

To cite: Moreno JP, VézinaIm L-A, Vaughan EM, et al. Impact of child summertime obesity interventions on body mass index, and weight-related behaviours: a systematic review and metaanalysis protocol. BMJ Open 2017;7:e017144. doi:10.1136/ bmjopen-2017-017144

- Prepublication history and additional material for this paper are available online. To view please visit the journal (http:// dx.doi.org/10.1136/bmjopen2017-017144).

Received 5 April 2017 Revised 24 August 2017 Accepted 4 September 2017

CrossMark

${ }^{1}$ Department of PediatricsNutrition, USDA/ARS Children's Nutrition Research Center, Baylor College of Medicine, Houston, Texas, USA

${ }^{2}$ Department of Medicine, Baylor College of Medicine, Houston, Texas, USA

Correspondence to Dr Jennette P Moreno; palcic@bcm.edu

\section{ABSTRACT}

Introduction In previous studies, it has been found that on average, children consistently gained weight during the summer months at an increased rate compared with the 9-month school year. This contributed to an increased prevalence of overweight and obesity in children. Several obesity-related interventions have occurred during or targeting the summer months. We propose to conduct a systematic review and meta-analysis of the impact of obesity prevention and treatment interventions for schoolage children conducted during the summer or targeting the summer months when children are not in school on their body mass index (BMI), or weight-related behaviours. Methods and analyses A literature search will be conducted by the first author (JPM) using MEDLINE/ PubMed, Cochrane Library, Scopus, CINAHL, PsycINF0, EMBASE and Proquest Dissertations and Theses databases from the date of inception to present. Studies must examine interventions that address the modification or promotion of weight-related behaviours (eg, dietary patterns, eating behaviours, physical activity (PA), sedentary behaviour or sleep) and target school-age children (ages 5-18). The primary outcomes will be changes from baseline to postintervention and/or the last available follow-up measurement in weight, BMl, BMI percentile, standardised BMI or per cent body fat. Secondary outcomes will include changes in dietary intake, PA, sedentary behaviour or sleep. Risk of bias will be assessed using the Cochrane risk of bias tool for randomised and non-randomised studies, as appropriate. Ethics and dissemination Because this is a protocol for a systematic review, ethics approval will not be required. The findings will be disseminated via presentations at scientific conferences and published in a peer-reviewed journal. All amendments to the protocol will be documented and dated and reported in the PROSPERO trial registry. PROSPERO registration number CRD42016041750

\section{INTRODUCTION}

Obesity is a critical public health problem in the USA with $17 \%$ of children and $38 \%$ of adults classified as obese or extremely obese. ${ }^{12}$ Globally, 73 countries have experienced a doubling in the prevalence of obesity

\section{Strengths and limitations of this study}

- This will be the first review to systematically examine the impact of behavioural obesity prevention and treatment interventions conducted during or targeting the summer months when children are not in school on changes in children's weight status and/or weight-related behaviours during summer. Results will help to inform future behavioural obesity prevention and treatment interventions for summertime.

- Since a limited number of studies may have delivered obesity prevention interventions during summer months, the scope of the review will also include obesity treatment interventions occurring during the summer break from school.

- In addition to objectively measured body composition outcomes, we will include studies assessing diet, physical activity, sedentary behaviour and sleep outcomes using self-reported or parent-reported measures. These measures are known to be subject to substantial error.

among children and adults since $1980 .^{3}$ Obesity increases the risk for diabetes, ${ }^{4}$ heart disease $^{4}$ and cancer. ${ }^{5}$ It is associated with all leading causes of preventable death ${ }^{6}$ and has a US estimated annual medical cost of US $\$ 149.4$ billion. ${ }^{7}$ In $2015,7 \%$ of all deaths globally (4.0 million) were associated with having a high body mass index (BMI). ${ }^{3}$ Cardiovascular disease and diabetes were the leading causes of obesity-related deaths worldwide. ${ }^{3}$ In the USA, rates of obesity appeared to be higher among school-age children with $17.5 \%$ of those aged $6-11$ years and $20.5 \%$ of those aged 12-19years being classified as obese compared with only $8.9 \%$ of those aged $2-5$ years, ${ }^{8}$ suggesting this is a time when increased focus on the prevention of weight gain is needed.

Findings from studies of two large nationally representative samples of children in 
the USA, ${ }^{9}{ }^{10}$ and one each in Texas, ${ }^{11}{ }^{12}$ Minnesota, ${ }^{13}$ Wyoming $^{14}$ and Osaka Prefecture and Tokyo, Japan ${ }^{15} 16$ consistently demonstrated that school-age children gained weight at an accelerated rate during the summer months. Summertime increases in standardised BMI contribute to increased risk for obesity, ${ }^{1718}$ especially among children at risk for developing chronic health conditions associated with obesity. ${ }^{18} \mathrm{~A}$ large 5-year longitudinal study that examined children's standardised BMI biannually for 5 years throughout elementary school showed that about $19 \%$ of children began a trajectory towards overweight or obesity during early elementary school when they were $5-8$ years. ${ }^{17}$ The greatest increases in risk for overweight and obesity occurred during summer vacation. ${ }^{1718}$

Less is known about summer weight gain among preschoolers, with one study in Japan failing to find evidence of summer weight gain until 5 years old ${ }^{15}$ and another demonstrating summer weight gain among overweight children enrolled in HeadStart (ie, early childhood education for children from low-income families) and healthy-weight children receiving Medicaid (ie, government-assisted health insurance for children from low-income families) in Michigan. ${ }^{19}$ Studies involving middle school students have yielded inconsistent findings. Primarily Caucasian middle schoolers in the US Pacific Northwest demonstrated improvements in their BMI during summer months. ${ }^{20}$ No significant change in BMI during summer was observed among American Indian middle schoolers in Wyoming. ${ }^{14}$ However, results from a randomised controlled trial (RCT) of an intensive school-based obesity intervention in Texas reported significant increases in standardised BMI among Hispanic adolescents randomised to both the treatment and control conditions, though summertime increases in standardised BMI were significantly less in the treatment condition. $^{21}$

Summer weight gain seems to affect school-age children regardless of race, ethnicity, weight status classification or socioeconomic status ${ }^{10}{ }^{11}$; however, some studies suggest that overweight children and adolescents, African Americans, Hispanics and children of low socioeconomic status may display greater rates of accelerated summer weight gain. ${ }^{12} 1822$ Further, there is evidence that improvements achieved by school-based obesity and fitness interventions are lost over the summer. ${ }^{23-26}$ While less is known about the influence of accelerated weight gain during out-ofschool periods globally, summer weight gain in school-age children may be significantly impeding progress towards addressing the global obesity pandemic in children, representing an important opportunity to address the problem. ${ }^{27}$

Previous investigations into the causes of summer weight gain have focused primarily on physical activity (PA) and to a lesser extent diet, screen media use or sleep. ${ }^{28}$ Several studies have demonstrated that children increase their activity levels during summer ${ }^{29-31}$ while others showed a decrease $^{13}$ or no differences in activity levels or energy expenditure compared with the school year. ${ }^{32}$ Three studies examined changes in diet during the school year and summer. One study found that children aged 9-11 years in Minnesota demonstrated no seasonal differences in kilocalories and macronutrient intake ${ }^{13}$ while another showed a summertime increase in total calories $(+82 \pm 31 \mathrm{kcal})$ among Greek children aged $3-18$ years. ${ }^{33}$ Children in the US National Health and Nutrition Examination Survey 2003-2008 aged 6-19 decreased their vegetable intake by 0.2 servings per day, increased their added sugar intake by 2.1 teaspoons per day during summer break compared with the school year and increased their screen media use by $18 \mathrm{~min}$ per day during summer. ${ }^{31}$ Finally, sleep duration among elementary school-age children has been demonstrated to be 40.5 min shorter during summer compared with winter, $31.1 \mathrm{~min}$ shorter compared with fall and 14.8 min compared with spring. ${ }^{34}$ Overall, relatively few studies have examined seasonal differences in weight-related behaviours and their association with seasonal weight change. Studies that have examined these associations have produced inconsistent findings. As a result, the weight-related behaviours that underlie summer weight gain and their relationship to weight change during the school year and summer are poorly understood.

Typically, observational studies are used to study seasonal differences in weight-related behaviours and their association with differences in seasonal weight gain. Obesity prevention and treatment studies experimentally manipulate weight-related behaviours, offering another opportunity to study the impact of changes in these behaviours on weight outcomes. Such studies may help identify important behavioural targets for the prevention and treatment of summer weight gain, behaviours amenable to change, effective intervention components and the level of BMI changed.

While few studies have examined determinants of summer weight gain, several obesity interventions have been conducted during summer. ${ }^{35-76}$ Summer obesity interventions are typically delivered via day camps ${ }^{35} 365657$ 62-64 71-73 or residential camps, ${ }^{37-3944} 495052-5456$ 65-69 but also include school-based interventions ${ }^{41}{ }^{43}$ and multilevel community-based interventions. ${ }^{6174-76}$ We are not aware of any systematic reviews or meta-analyses focusing specifically on the impact of summertime childhood obesity prevention and treatment interventions on BMI, dietary intake, PA, screen media use or sleep. We propose to conduct a systematic review and meta-analysis of interventions for obesity prevention and treatment among school-age children conducted during or targeting the summer months when children are not in school, and assessing their impact on BMI, diet, PA, screen media use and sleep. This systematic review will examine (1) the impact of behavioural obesity prevention interventions on children's weight status and/or weight-related behaviours during summer, (2) whether the effects of the interventions varied by the behaviours targeted or behavioural intervention techniques employed ${ }^{77}$ and (3) biases in included studies. 


\section{METHODS}

\section{Registration}

This protocol has been registered with PROSPERO (registration number CRD42016041750) and reported in accordance with Preferred Reporting Items for Systematic Reviews and Meta-Analyses (PRISMA) ${ }^{78}$ PRISMA-Protocol $^{79}$ and PRISMA-Protocol for Children Extension ${ }^{80}$ guidelines.

\section{Study selection criteria}

Interventions and population

Studies reporting results of behavioural obesity prevention and treatment interventions occurring during or targeting the summer season and/or summer break from school will be included. Behavioural obesity prevention interventions will be defined as those targeting weight-related behaviours (eg, dietary patterns, eating behaviours, PA, screen media use, sedentary behaviour and sleep) among school-age children (ages 5-18). The summer season or summer break from school for studies conducted within the USA will include the following months: June, July and August. For studies conducted in the southern hemisphere, summer will be considered December, January and February, though for all studies occurring outside of the USA, we will research when the typical break from school occurs in that particular country.

\section{Outcomes}

Outcomes must include one or more of the following: changes in objective or self-reported measurement of weight, BMI, BMI percentile, standardised BMI, per cent body fat, waist circumference, dietary intake, PA, screen media use, sedentary behaviour or sleep from baseline to postintervention and the last available follow-up measurement (table 1). Studies will be grouped for meta-analysis according to the outcome(s) provided.

Table 1 Outcomes and operational definitions

\section{Operational definitions}

Primary outcomes*

Body weight

Body mass index (BMI)

BMI percentile

Standardised BMI

Per cent body fat

Waist circumference

Secondary outcomes*

Dietary intake

Physical activity and sedentary behaviour

Screen media use

Night-time sleep duration
We will include studies that measured body weight using an objective measure of body weight in kilograms measured using a scale (eg, digital scale, beam scale). If body weight was reported in pounds, it will be converted to kilograms.

For studies reporting BMI outcomes, we will only include studies where BMI was calculated using objectively measured height and weight, and one of the following formulas: weight $(\mathrm{kg}) /(\text { height }(\mathrm{cm}))^{2}$ or weight (lbs)/(height (inches)) $)^{2} \times 703$.

We will include studies that measured BMI percentile calculated based on growth charts from the Centers for Disease Control and Prevention (CDC) or WHO. $^{83}$

We will include studies in which BMI was standardised using age and gender normative data from the $\mathrm{CDC}^{84}$ or the WHO references. ${ }^{85}$

We will include studies in which per cent body fat was measured by bioelectrical impedance or dual-energy X-ray absorptiometry.

We will include studies in which the circumference ${ }^{86}$ of the waist was measured at the level of the belly button. If waist circumference was reported in inches, it will be converted to centimetres.

We will include studies examining three primary diet-related outcomes: total calories consumed, servings or cups of fruit and vegetables, and servings of sugar-sweetened beverages. We will also record other obesity-related dietary outcomes and patterns assessed (eg, skipping breakfast, snacking frequency, late-night snacking and eating meals at routine times) and we will summarise these findings.

We will examine studies reporting the number of minutes per day of moderate-intensity to vigorous-intensity physical activity and sedentary behaviour measured via accelerometry or self-report/parent-report measures or diaries.

We will include studies reporting the number of minutes per day of screen media use (eg, television, mobile electronic devices, video games and computers) as measured by selfreport/parent-report measures or diaries.

We will include studies reporting total night-time sleep duration as the total number of hours and minutes of sleep from bedtime to wake time as assessed via actigraphy or parentreport/self-report. Naps will not be included in total night-time sleep duration. We will also record other sleep-related outcomes (ie, sleep duration variability, bedtime and sleep quality) and we will summarise these findings.

*We will examine change in these outcomes at post-intervention and the last reported follow-up. 


\section{Study designs}

RCTs, quasi-experimental RCTs, and controlled or uncontrolled before-and-after comparisons will be included to allow for the inclusion of pilot studies. Studies using a waitlist, usual care, no-treatment or alternative treatment controls will be included.

\section{Other}

Studies must be published in English, French or Spanish, with no restriction on the year of publication.

\section{Exclusion criteria}

Studies will be excluded for the following reasons: (1) participants were diagnosed with pre-existing medical conditions (eg, diabetes, non-alcoholic fatty liver disease, cancer); (2) active intervention phase of less than 1 day; (3) letters to the editor, meta-analyses, case reports and reviews; or (4) abstracts and conference proceedings.

\section{Search strategy}

A literature search will be conducted by the first author (JPM) using MEDLINE/PubMed, Cochrane Library, Scopus, CINAHL, PsycINFO and EMBASE databases from the date of inception to present. Proquest Dissertations and Theses will also be explored for unpublished trials (a form of grey literature). Structured search strings will be developed using the following key words: (1) summer or seasonal; (2) children or adolescents or kid or boy or girl; (3) overweight, obese, obesity, or weight gain, weight, body mass index, BMI, dietary intake, diet, PA, sleep, sedentary behavior, screen media use, television, $\mathrm{TV}$, video game use, computer use, mobile phone, body fat, adiposity or weight status; (4) intervention, program, prevention, or treatment. Additional studies will be gleaned from the reference lists of studies chosen for inclusion in the systematic review. Search strategy results will be reported using a PRISMA flow diagram. ${ }^{81} \mathrm{~A}$ draft of the search strategy is provided in online supplementary file 1 .

\section{Study selection}

Initial title and abstract review will be conducted by the first author (JPM). Duplicates and articles clearly not meeting selection criteria will be removed. The remaining list will be retrieved and two reviewers (JPM and EMV) will independently review the retrieved articles for eligibility. Overall agreement rate will be calculated using Cohen's $\kappa$. A third reviewer (LAVI) will be consulted in situations when agreement is not achieved. In the event that multiple studies reporting results of the same intervention with the same sample are identified, reviewers will attempt to verify if this is the case by contacting the authors, and preference for inclusion will be given to studies reporting changes in outcomes from baseline to postintervention and follow-up and studies using more rigorous methods.

\section{Data extraction}

Codebooks adapted from the Cochrane data collection form for intervention reviews will be developed in Excel and piloted by two reviewers using five manuscripts. Two reviewers (JPM and EMV) will meet to discuss discrepancies and propose any needed changes to the codebooks.

Using the refined codebooks, data will be abstracted independently by two reviewers (JPM and EMV). Information will include setting, location of intervention delivery (eg, day camp, school, community, residential camp), study design, country, participant recruitment, participant recruitment sources, inclusion and exclusion criteria, participant allocation, sample size, demographics of the sample, intervention components, ${ }^{77}$ intervention duration, theoretical framework, dose of intervention delivered (duration in minutes, length of the intervention in days or weeks and frequency of contact), treatment fidelity, treatment compliance, outcome measurements, time points measured, means and SD for continuous outcomes measured, and handling of missing data. Codebooks created independently by reviewers will be merged and reviewers will meet to review for agreement. Discrepancies will be resolved by discussion and a third party (LAVI) will be consulted as needed.

\section{Outcomes and prioritisation}

The primary outcome will include changes in weight status-related measures including weight, BMI, BMI percentile, ${ }^{82} 83$ standardised BMI, ${ }^{84}$ per cent body fat and waist circumference. ${ }^{86}$ Preference will be given for standardised BMI and if not available, the authors will attempt to calculate standardised BMI by using available data or data made available to the authors. In the event that we are unable to convert data to standardised BMI, outcomes will be grouped according to the outcome measure collected. Secondary outcomes will include changes in targeted behaviour such as dietary intake, PA, screen media use, sedentary behaviour or sleep.

\section{Risk of bias and quality assessments}

Risk of bias will be assessed independently by JPM and EMV using the Cochrane risk of bias tool for randomised and non-randomised studies, as appropriate. ${ }^{87}$ The Cochrane risk of bias tool for RCTs considers the following criteria: (1) randomisation sequence generation: was the allocation sequence adequately generated?; (2) treatment allocation concealment: was the allocated treatment adequately concealed from study participants and clinicians and other healthcare or research staff at the enrolment stage?; (3) blinding: were participants and research staff blind to treatment allocation?; (4) blinding: were the personnel assessing outcomes and analysing data sufficiently blinded to the intervention allocation throughout the trial?; (5) completeness of outcome data: were participant exclusions, attrition and incomplete outcome data adequately addressed in the published report?; (6) selective outcome reporting: is there evidence of selective outcome reporting and might this have affected the study 
results?; (7) other sources of bias: was the trial apparently free of any other problems that could produce a high risk of bias?. ${ }^{87}$ Questions are rated as having a high, low or unclear level of bias across the seven domains.

The Cochrane risk of bias tool for non-randomised studies of interventions considers risk of bias across three domains: (1) preintervention (ie, potential confounders and selection of participants), (2) intervention (ie, classification of interventions) and (3) postintervention (ie, protocol deviations, missing data, measurement of outcomes and selective reporting of results). Studies are rated as having a low, moderate, serious and critical risk for bias. Discrepancies in ratings will be discussed until consensus is achieved or a third party (LAVI) will be consulted. Questions are rated as having a critical, moderate, low or unclear level of bias across the three domains.

The Grading Quality of Evidence and Strength of Recommendations (GRADE) guidelines will be used to assess the quality of the evidence. ${ }^{88}$ GRADE addresses methodological flaws, consistency of results across studies, generalisability of results to the target population and effect size. Each quality outcome is rated as high, moderate, low or very low.

\section{Data management and statistical analyses}

All articles retrieved from the search will be imported into EndNote X7. ${ }^{89}$ Data obtained through data extraction will be entered into Excel. Outcomes will be imported into Comprehensive Meta-analysis software ${ }^{90}$ for meta-analysis.

We will create a table describing study characteristics and major outcomes. We will provide a narrative summary of the results from the included studies, organised by type of intervention (ie, prevention or treatment), treatment modality (ie, day or residential camp, community based, school based or clinic based), targeted behaviours (ie, dietary patterns and intake, PA, sleep, sedentary behaviour), target population characteristics (eg, ethnicity, sex, age, culture), geographic location of the study, type of outcome and intervention content. Intervention effects for each study will be calculated using standardised mean differences (for continuous outcomes). We will stratify studies according to whether they are obesity treatment or prevention interventions. Studies involving the same type of intervention, treatment modality, comparator and outcome measure will be grouped.

Results will be pooled using a random-effects meta-analysis, with standardised mean differences for continuous outcomes, and we will calculate $95 \%$ CIs and two-sided $\mathrm{p}$ values for each outcome. For studies that have not accounted for the effects of clustering, SD will be adjusted for the design effect. Heterogeneity between the studies in effect measures will be assessed using both the $\chi^{2}$ test and the $\mathrm{I}^{2}$ statistic. ${ }^{91}$ We will consider an $\mathrm{I}^{2}$ value greater than $75 \%$ is indicative of substantial heterogeneity. ${ }^{92}$ When a meta-analysis of results is not appropriate, a narrative summary of results will be provided. We will conduct sensitivity analyses to explore heterogeneity in effect estimates according to participant characteristics (eg, gender, age, weight status), location, theoretical basis of the intervention (eg, theory vs non-theory based), behaviours targeted (eg, diet only, PA only, diet and PA, screen media use, sleep), intervention components, ${ }^{77}$ risk of bias and study quality. If our meta-analysis contains greater than 10 studies, a funnel plot analysis will be performed to assess for publication bias and a visual inspection for asymmetry will be performed ${ }^{93}$ and by using Egger's ${ }^{94}$ regression test for continuous variables.

\section{DISCUSSION}

This systematic review and meta-analysis will be the first to examine the efficacy and effectiveness of behavioural summer obesity prevention and treatment interventions to reduce weight outcomes among school-age children during the summer months when children are not in school and the impact on targeted behavioural outcomes (eg, diet, PA, screen media use, sedentary behaviour and sleep). Secondary analyses will examine differences in outcomes according to participant characteristics such as gender, age, weight status, location, theoretical basis of the intervention, intervention components ${ }^{77}$ and quality of studies. By evaluating the impact of obesity interventions on weight outcomes, we hope to identify the overall efficacy and effectiveness of interventions conducted during the summer or targeting summer weight gain.

A previous review examined non-curricular approaches to increasing PA, including summer day camps and active travel to school, extracurricular activities and interventions delivered during school break periods (eg, recess). Their review of summer day camps found that day camps were effective in increasing PA, though further research was needed regarding the promotion of long-term habitual physical activity. ${ }^{59} \mathrm{~A}$ similar review examined the impact of obesity prevention and treatment interventions conducted during out-of-school times (eg, after school and summertime) among African American youth and identified only one summertime obesity prevention intervention, which demonstrated a trend towards reduced BMI ${ }^{58}$ While we are primarily interested in the impact of obesity prevention interventions on summer weight gain, there are likely too few such studies to warrant a review. Thus, the focus of this review was expanded to examine the prevention of obesity in adulthood and, therefore, obesity treatment interventions. A review of immersion programmes, many of which occurred during the summer break from school, found immersion programmes produced significantly greater weight loss compared with outpatient interventions. These findings suggested immersion programmes were a promising method of intervening with youth. ${ }^{60}$ The current review will build on the previous findings, focusing specifically on summer as a time to prevent or treat obesity in children, and will examine the impact of the interventions on weight outcomes and weight-related behaviours. 
Examination of the impact of the interventions on weight-related behaviours will identify important behavioural targets for the prevention of summer weight gain and effective strategies for inducing behaviour change. Results will help to inform factors associated with summer weight gain in children as well as refinement and future directions for development of behavioural obesity prevention interventions for summertime. Prevention of summer weight gain is an important opportunity to address the worldwide childhood obesity epidemic.

We would like to acknowledge some of the likely limitations of this review. First, while objective measures of outcomes are preferred, we will include studies assessing secondary outcomes using self-reported or parent-reported behavioural weight-related outcomes. Self-report or parent reports of diet, PA and sleep are known to overestimate sleep ${ }^{95} 96$ and $\mathrm{PA}^{97}$ and underestimate dietary intake. ${ }^{98}$ Second, it is challenging to group interventions together even by type and modality of intervention and to determine the impact of the intervention as methods and outcome measures may vary greatly across studies. As such, we will stratify studies according to whether they are obesity treatment or prevention interventions and group them according to treatment modality, comparator and outcome measure. Finally, this review may be limited by the number of summertime interventions fitting into these categories. In the event that an insufficient number of studies are identified for performing a meta-analysis, we will provide a narrative review of the studies and their outcomes.

Contributors JPM, the guarantor of the protocol, drafted the protocol and registered it in PROSPERO. TB reviewed and commented on the protocol in PROSPERO. JPM, EMV, LAVI and TB all reviewed and commented on this protocol.

Funding This work is a publication of the USDA (USDA/ARS) Children's Nutrition Research Center, Department of Pediatrics, Baylor College of Medicine (Houston, TX) and has had been funded, in part, with federal funds from the USDA/ARS under Cooperative Agreement Number 58-6250-6001. JPM is the recipient of grants from the Boyd and Evelyn Mullen Foundation and the Texas Children's Hospital Pediatric Pilot Research Fund, which played no role in the development or review of the protocol.

Competing interests None declared.

Provenance and peer review Not commissioned; externally peer reviewed.

Open Access This is an Open Access article distributed in accordance with the Creative Commons Attribution Non Commercial (CC BY-NC 4.0) license, which permits others to distribute, remix, adapt, build upon this work non-commercially, and license their derivative works on different terms, provided the original work is properly cited and the use is non-commercial. See: http://creativecommons.org/ licenses/by-nc/4.0/

(c) Article author(s) (or their employer(s) unless otherwise stated in the text of the article) 2017. All rights reserved. No commercial use is permitted unless otherwise expressly granted.

\section{REFERENCES}

1. Ogden CL, Carroll MD, Lawman HG, et al. Trends in obesity prevalence among children and adolescents in the United States, 1988-1994 through 2013-2014. JAMA 2016;315:2292-9.

2. Flegal KM, Kruszon-Moran D, Carroll MD, et al. Trends in obesity among adults in the United States, 2005 to 2014. JAMA 2016;315:2284-91.
3. Afshin A, Forouzanfar MH, Reitsma MB, et al. Health effects of overweight and obesity in 195 countries over 25 years. N Engl J Med 2017;377.

4. Goran MI, Ball GD, Cruz ML. Obesity and risk of type 2 diabetes and cardiovascular disease in children and adolescents. J Clin Endocrinol Metab 2003;88:1417-27.

5. De Pergola G, Silvestris F. Obesity as a major risk factor for cancer. J Obes 2013;2013:1-11.

6. Yoon PW, Bastian B, Anderson RN, et al. Potentially preventable deaths from the five leading causes of death-United States, 2008-2010. MMWR Morb Mortal Wkly Rep 2014;63:369-74.

7. Kim DD, Basu A. Estimating the medical care costs of obesity in the United States: systematic review, meta-analysis, and empirical analysis. Value Health 2016;19:602-13.

8. Ogden CL, Carroll MD, Kit BK, et al. Prevalence of childhood and adult obesity in the United States, 2011-2012. JAMA 2014;311:806-14.

9. von Hippel PT, Powell B, Downey DB, et al. The effect of school on overweight in childhood: gain in body mass index during the school year and during summer vacation. Am J Public Health 2007;97:696-702.

10. von Hippel PT, Workman J. From kindergarten through second grade, U.S. children's obesity prevalence grows only during summer vacations. Obesity 2016;24:2296-300.

11. Moreno JP, Johnston CA, Chen TA, et al. Seasonal variability in weight change during elementary school. Obesity 2015;23:422-8.

12. Moreno JP, Johnston CA, Woehler D. Changes in weight over the school year and summer vacation: results of a 5-year longitudinal study. J Sch Health 2013;83:473-7.

13. McCue MC, Marlatt KL, Sirard J, et al. Examination of changes in youth diet and physical activity over the summer vacation period. Internet J Allied Health Sci Prac 2013;11:1-6.

14. Smith DT, Bartee RT, Dorozynski CM, et al. Prevalence of overweight and influence of out-of-school seasonal periods on body mass index among American Indian school children. Prev Chronic Dis 2009;6:A20.

15. Kato N, Sauvaget $C$, Kato $T$. Large summer weight gain in relatively overweight preschool Japanese children. Pediatr Int 2012;54:510-5.

16. Kobayashi $\mathrm{M}$, Kobayashi $\mathrm{M}$. The relationship between obesity and seasonal variation in body weight among elementary school children in Tokyo. Econ Hum Biol 2006;4:253-61.

17. Chen TA, Baranowski T, Moreno JP, et al. Obesity status trajectory groups among elementary school children. BMC Public Health 2016;16:526.

18. Chen TA, Baranowski T, Moreno JP, et al. Obesity status transitions across the elementary years: use of Markov chain modelling. Pediatr Obes 2016;11:88-94.

19. Lumeng JC, Kaciroti N, Sturza J, et al. Changes in body mass index associated with head start participation. Pediatrics 2015;135:e44 9-e456.

20. Daratha KB, Power TG, Steele MM, et al. Changes in BMI between summer and school months among middle school students. Washington State Journal of Public Health Practice 2009;2:13-21.

21. Reesor L, Hernandez D, Johnston CA, et al. School-based weight management program curbs summer weight gain among low-income Hispanic middle school students. under review.

22. Franckle R, Adler R, Davison K. Accelerated weight gain among children during summer versus school year and related racial/ethnic disparities: a systematic review. Prev Chronic Dis 2014;11:E101.

23. Gutin B, Yin Z, Johnson M, et al. Preliminary findings of the effect of a 3-year after-school physical activity intervention on fitness and body fat: the Medical College of Georgia Fitkid Project. Int J Pediatr Obes 2008;3(Suppl 1):3-9.

24. Yin Z, Moore JB, Johnson MH, et al. The impact of a 3-year afterschool obesity prevention program in elementary school children. Child Obes 2012;8:60-70.

25. Carrel AL, Clark RR, Peterson S, et al. School-based fitness changes are lost during the summer vacation. Arch Pediatr Adolesc Med 2007;161:561-4.

26. Gillis L, McDowell M, Bar-Or O. Relationship between summer vacation weight gain and lack of success in a pediatric weight control program. Eat Behav 2005;6:137-43.

27. Brown CL, Halvorson EE, Cohen GM, et al. Addressing childhood obesity: opportunities for prevention. Pediatr Clin North Am 2015;62:1241-61.

28. Baranowski T, O'Connor T, Johnston $\mathrm{C}$, et al. School year versus summer differences in child weight gain: a narrative review. Child Obes 2014;10:18-24.

29. Rich C, Griffiths LJ, Dezateux C. Seasonal variation in accelerometerdetermined sedentary behaviour and physical activity in children: a review. Int J Behav Nutr Phys Act 2012;9:49. 
30. Bruner MW, Chad KE, Beattie-Flath JA, et al. Examination of physical activity in adolescents over the school year. Pediatr Exerc Sci 2009;21:421-35.

31. Wang YC, Vine S, Hsiao A, et al. Weight-related behaviors when children are in school versus on summer breaks: does income matter? J Sch Health 2015;85:458-66.

32. Zinkel SR, Moe M, Stern EA, et al. Comparison of total energy expenditure between school and summer months. Pediatr Obes 2013;8:404-10.

33. Yannakoulia M, Drichoutis AC, Kontogianni MD, et al. Season-related variation in dietary recalls used in a paediatric population. $J$ Hum Nutr Diet 2010;23:489-93.

34. Nixon GM, Thompson JM, Han DY, et al. Short sleep duration in middle childhood: risk factors and consequences. Sleep 2008;31:71-8.

35. Baranowski T, Baranowski JC, Cullen KW, et al. The fun, food, and fitness project (FFFP): the Baylor GEMS pilot study. Ethn Dis 2003;13(1 Suppl 1):S30-9

36. Burke SM, Shapiro S, Petrella RJ, et al. Using the RE-AIM framework to evaluate a community-based summer camp for children with obesity: a prospective feasibility study. BMC Obes 2015;2:21.

37. Gately PJ, Cooke CB, Barth JH, et al. Children's residential weight-loss programs can work: a prospective cohort study of short-term outcomes for overweight and obese children. Pediatrics 2005;116:73-7.

38. Gately PJ, Cooke CB, Butterly RJ, et al. The effects of a children's summer camp programme on weight loss, with a 10 month followup. Int J Obes Relat Metab Disord 2000;24:1445-52.

39. Huelsing J, Kanafani N, Mao J, et al. Camp jump start: effects of a residential summer weight-loss camp for older children and adolescents. Pediatrics 2010;125:e884-e890.

40. Kellam S, Olvera N, Leung P, et al. Effect of exercise dosages on adiposity indices in overweight girls. Salud Publica Mex 2013;55(Suppl 3):415-21.

41. Kilanowski JF, Gordon NH. Making a difference in migrant summer school: testing a healthy weight intervention. Public Health Nurs 2015;32:421-9.

42. Kotler LA, Etu SF, Davies M, et al. An open trial of an intensive summer day treatment program for severely overweight adolescents. Eat Weight Disord 2006;11:e119-e122.

43. Lloyd JJ, Wyatt KM, Creanor S. Behavioural and weight status outcomes from an exploratory trial of the Healthy Lifestyles Programme (HeLP): a novel school-based obesity prevention programme. BMJ Open 2012;2:e000390.

44. Meucci M, Cook C, Curry CD, et al. Effects of supervised exercise program on metabolic function in overweight adolescents. World $J$ Pediatr 2013;9:307-11.

45. Olvera N, Leung P, Kellam SF, et al. Body fat and fitness improvements in Hispanic and African American girls. $J$ Pediatr Psychol 2013;38:987-96.

46. Olvera N, Leung P, Kellam SF, et al. Summer and follow-up interventions to affect adiposity with mothers and daughters. Am J Prev Med 2013;44(3 Suppl 3):S258-S266.

47. Olvera N, Scherer R, McLeod J, et al. BOUNCE: an exploratory healthy lifestyle summer intervention for girls. Am J Health Behav 2010;34:144-55.

48. Roriz Oliveira MS, Seabra A, Maia J. Effects of a recreational-based physical activity summer camp on body composition, metabolic syndrome and physical fitness in obese children: a pilot study. J Sports Med Phys Fitness 2015.

49. Tang $\mathrm{Q}$, Ruan $\mathrm{H}$, Tao $\mathrm{Y}$, et al. Effects of a summer program for weight management in obese children and adolescents in Shanghai. Asia Pac J Clin Nutr 2014;23:459-64.

50. Valrie CR, Bond K, Lutes LD, et al. Relationship of sleep quality, baseline weight status, and weight-loss responsiveness in obese adolescents in an immersion treatment program. Sleep Med 2015;16:432-4.

51. Wang CL, Liang L, Fu JF, et al. Effect of lifestyle intervention on non-alcoholic fatty liver disease in Chinese obese children. World $J$ Gastroenterol 2008;14:1598-602.

52. Wong WW, Abrams SH, Mikhail C, et al. An innovative summer camp program improves weight and self-esteem in obese children. $J$ Pediatr Gastroenterol Nutr 2009;49:493-7.

53. Wong WW, Barlow SE, Mikhail C, et al. A residential summer camp can reduce body fat and improve health-related quality of life in obese children. J Pediatr Gastroenterol Nutr 2013;56:83-5.

54. Zou ZC, -J Mao L, Shi YY, et al. Effect of exercise combined with dietary intervention on obese children and adolescents associated with the FTO rs9939609 polymorphism. Eur Rev Med Pharmacol Sci 2015;19:4569-75.
55. Bohnert AM, Ward AK, Burdette KA, et al. Active summers matter: evaluation of a community-based summertime program targeting obesogenic behaviors of low-income, ethnic minority girls. New Dir Youth Dev 2014:2014:133-50.

56. Jirapinyo $\mathrm{P}$, Limsathayourat $\mathrm{N}$, Wongarn $\mathrm{R}$, et al. A summer camp for childhood obesity in Thailand. J Med Assoc Thai 1995;78:238-46.

57. Hopkins LC, Fristad M, Goodway JD, et al. Camp NERF: methods of a theory-based nutrition education recreation and fitness program aimed at preventing unhealthy weight gain in underserved elementary children during summer months. BMC Public Health 2016;16:1122.

58. Barr-Anderson DJ, Singleton C, Cotwright CJ, et al. Outside-ofschool time obesity prevention and treatment interventions in African American youth. Obes Rev 2014;15(Suppl 4):26-45.

59. Jago R, Baranowski T. Non-curricular approaches for increasing physical activity in youth: a review. Prev Med 2004;39:157-63.

60. Kelly KP, Kirschenbaum DS. Immersion treatment of childhood and adolescent obesity: the first review of a promising intervention. Obes Rev 2011;12:37-49.

61. Pierce B, Bowden B, McCullagh M, et al. A summer health program for African-American high school students in Baltimore, Maryland: community partnership for integrative health. Explore 2017:13:186-97.

62. Roriz DE Oliveira MS, Teixeira Seabra AF, Ribeiro Maia JA. Effects of a recreational physical activity summer camp on body composition, metabolic syndrome and physical fitness in obese children. $J$ Sports Med Phys Fitness 2016;56:933-8.

63. Bates CR, Bohnert AM, Ward AK, et al. Sleep is in for summer: patterns of sleep and physical activity in urban minority girls. $J$ Pediatr Psychol 2016;41:692-700.

64. Weaver RG, Brazendale K, Chandler JL, et al. First year physical activity findings from turn up the HEAT (Healthy Eating and Activity Time) in summer day camps. PLoS One 2017:12:e0173791.

65. Jennings L, Nepocatych S, Ketcham C, et al. The effect of a summer camp intervention on the nutrition knowledge and dietary behavior of adolescent girls. Health Promot Pract 2016;17:521-9.

66. Carraway ME, Lutes LD, Crawford Y, et al. Camp-based immersion treatment for obese, low socioeconomic status, multi-ethnic adolescents. Child Obes 2014;10:122-31.

67. Sampat S, Kirschenbaum DS, Gierut KJ, et al. Ya gotta have friends: social support and self-efficacy predict success following immersion treatment. Obesity 2014;22:2579-85.

68. Farhat AE, Sharma S, Abrams SH, et al. Kamp K'aana, a 2-week residential weight management summer camp, shows long-term improvement in body mass index z scores. J Pediatr Gastroenterol Nutr 2016;62:491-4.

69. Fonseca H, Palmeira AL, Martins S, et al. Short- and medium-term impact of a residential weight-loss camp for overweight adolescents. Int J Adolesc Med Health 2014;26:33-8.

70. Hickerson BD, Henderson KA. Opportunities for promoting youth physical activity: an examination of youth summer camps. J Phys Act Health 2014:11:199-205.

71. Baranowski T, Jago R. Understanding the mechanisms of change in children's physical activity programs. Exerc Sport Sci Rev 2005;33:163-8.

72. Southam MA, Kirkley BG, Murchison A, et al. A summer day camp approach to adolescent weight loss. Adolescence 1984;19:855-68.

73. George GL, Schneider C, Kaiser L. Healthy lifestyle fitness camp: a summer approach to prevent obesity in low-income youth. $J$ Nutr Educ Behav 2016:48:208-12.

74. Alfonso ML, McDermott RJ, Thompson Z, et al. Vigorous physical activity among tweens, VERB summer scorecard program, Lexington, Kentucky, 2004-2007. Prev Chronic Dis 2011;8:A104.

75. Alfonso ML, Thompson Z, McDermott RJ, et al. VERB TM Summer Scorecard: increasing tween girls' vigorous physical activity. J Sch Health 2013;83:164-70.

76. McDermott RJ, Davis JL, Bryant CA, et al. Increasing physical activity in children 8 to 12 years old: experiences with VERB Summer Scorecard. Percept Mot Skills 2010;111:240-8.

77. Michie S, Wood CE, Johnston M, et al. Behaviour change techniques: the development and evaluation of a taxonomic method for reporting and describing behaviour change interventions (a suite of five studies involving consensus methods, randomised controlled trials and analysis of qualitative data). Health Technol Assess 2015;19:1-188.

78. Moher D, Liberati A, Tetzlaff J, et al. Preferred reporting items for systematic reviews and meta-analyses: the PRISMA statement. PLoS Med 2009;6:e1000097.

79. Moher D, Shamseer L, Clarke M, et al. Preferred reporting items for systematic review and meta-analysis protocols (PRISMA-P) 2015 statement. Syst Rev 2015;4:1. 
80. Kapadia MZ, Askie L, Hartling L, et al. PRISMA-Children (C) and PRISMA-Protocol for Children (P-C) extensions: a study protocol for the development of guidelines for the conduct and reporting of systematic reviews and meta-analyses of newborn and child health research. BMJ Open 2016;6:e010270.

81. Moher D, Liberati A, Tetzlaff $\mathrm{J}$, et al. Preferred reporting items for systematic reviews and meta-analyses: the PRISMA statement. BM 2009:339.

82. CDC. CDC growth charts. 2000 http://www.cdc.gov/growthcharts/ (accessed 26 Jun 2017)

83. WHO. The WHO child growth standards website. $2006 \mathrm{http}: / / w w w$. who.int/childgrowth/en/ (accessed 26 Jun 2017).

84. Kuczmarski RJ, Ogden CL, Guo SS, et al. 2000 CDC Growth Charts for the United States: methods and development. Vital Health Stat 11 2002:2002:1-190.

85. WHO. WHO child growth standards: methods and development: growth velocity based on weight length and head circumference. 2009 http://www.who.int/childgrowth/standards/velocity/technical report/en/ (accessed 26 Jun 2017).

86. Colleran K, Harding E, Kipp BJ, et al. Building capacity to reduce disparities in diabetes: training community health workers using an integrated distance learning model. Diabetes Educ 2012;38:386-96.

87. Higgins JP, Altman DG, Gøtzsche PC, et al. The Cochrane Collaboration's tool for assessing risk of bias in randomised trials. BMJ 2011;343:d5928.

88. Atkins $D$, Best D, Briss PA, et al. Grading quality of evidence and strength of recommendations. BMJ 2004;328:1490.
89. EndNote X7 [computer program]. Version X7.4 Thomson Reuters. 2016.

90. Borenstein M, Hedges L, Higgins J, et al. Comprehensive metaanalysis, version 3. Englewood, NJ: Biostat, 2014

91. Higgins JP, Thompson SG. Quantifying heterogeneity in a metaanalysis. Stat Med 2002;21:1539-58.

92. Higgins JP, Thompson SG, Spiegelhalter DJ. A re-evaluation of random-effects meta-analysis. J $R$ Stat Soc Ser A Stat Soc 2009;172:137-59.

93. Sutton AJ, Duval SJ, Tweedie RL, et al. Empirical assessment of effect of publication bias on meta-analyses. BMJ 2000;320:1574-7.

94. Egger M, Davey Smith G, Schneider M, et al. Bias in meta-analysis detected by a simple, graphical test. BMJ 1997;315:629-34.

95. Markovich AN, Gendron MA, Corkum PV. Validating the children's sleep habits questionnaire against polysomnography and actigraphy in school-aged children. Front Psychiatry 2014;5:188.

96. Duraccio KM, Carbine KA, Barnett KA, et al. The utility of the children's sleep habits questionnaire: associations between parental report and an objective measure of sleep behavior. Children's Health Care 2017;23:1-17.

97. Adamo KB, Prince SA, Tricco AC, et al. A comparison of indirect versus direct measures for assessing physical activity in the pediatric population: a systematic review. Int $J$ Pediatr Obes 2009;4:2-27.

98. Livingstone MB, Robson PJ. Measurement of dietary intake in children. Proc Nutr Soc 2000;59:279-93. 